\title{
A biomimética no desenvolvimento de produtos: a relação entre forma e função para obtenção de leiautes iniciais
}

Biomimetics in products development: the relationship between shape and function for getting initial layouts

André Ogliari ${ }^{1}$

Leonardo Menezes Melo 


\section{Resumo}

O presente trabalho possui como objetivo apresentar de maneira geral como é realizado o processo de desenvolvimento de produtos, com foco na configuração da forma de soluções conprocesso propõe-se uma sistemática para a transição da fase de projeto conceitual para a de projeto preliminar, ou seja, para o desenvolvimento do leiaute inicial das soluções conceituais desenvolvidas. Após uma revisão bibliográfica sobre desenvolvimento de produtos e biomimética apresenta-se a proposta de uma matriz (Matriz FNF) que relaciona funções e formas baseadas em analogias com a natureza, visando oferecer uma alternativa às equipes para a configuração de forma nos elementos da solução conceitual desenvolvida. A matriz FNF é constituída de conteúdos e exemplos da natureza associado com funções técnicas de produtos, servindo como meio de inspiração para a geração de forma. Ao final são apresentados detalhes da referida matriz e exemplos parciais de sua utilização baseado em atividades para a geração do leiaute inicial do produto.

Palavras-Chave: Desenvolvimento de produtos; biomimética; forma; função. ceituais por meio da Biomimética. Nesse

\section{Abstract}

This work aims to present on a general view how the process of product development is carried out, with a focus on setting the shape of conceptual solutions through Biomimicry. It is proposed a systematic for the transition from conceptual design phase to the preliminary design, i.e, to the development of the initial layout of the developed conceptual solutions. After a literature review on product development and biomimetic the paper presents the proposal of a matrix (FNF Matrix) that lists functions and shape based on analogies with nature, in order to offer an alternative to the teams for the configuration shape of the elements of conceptual solution developed. The FNF matrix is comprised of contents and examples of the technical features associated with nature products, serving as inspirating means to shape generation. At the end it presents details of this matrix and partial examples of its use based on activities to generate the initial layout of the product.

Keywords: Products development; biomimetics; shape; function.

ISSN: 1808-3129

\footnotetext{
${ }^{1}$ Mestrando em Engenharia Mecânica pela Universidade Federal de Santa Catarina - UFSC leonardommelo@hotmail.com ${ }^{2}$ Doutor em Engenharia Mecânica pela Universidade Federal de Santa Catarina - UFSC andre.ogliari@ufsc.br
}

Artigo indicado através da organização do evento IDEMi 2015 


\section{INTRODUÇÃO}

Os profissionais que trabalham com o desenvolvimento de produtos têm buscado recursos com o intuito de aprimorar cada vez mais o processo como um todo, com o objetivo principal de obter melhores soluções em diferentes aspectos, como: redução de custos, maior satisfação dos clientes, além de conquistar o maior percentual do mercado atuante.

Com o propósito de aprimorar as etapas do processo de desenvolvimento de produtos, um tema que vem ganhando destaque é a biomimética.

A biomimética, de maneira geral, busca em conteúdo da natureza inspiração e soluções otimizadas para projetos de diferentes áreas do conhecimento. A área do conhecimento a que se aplica a biomimética neste trabalho é o do desenvolvimento de produtos no campo da engenharia.

O enfoque da pesquisa situa-se entre as etapas de projeto conceitual e projeto preliminar, e a proposta em questão de uso da biomimética leva em consideração a elaboração de uma matriz morfológica para auxiliar os profissionais na busca de situações presente na natureza para auxiliar na configuração da forma do produto.

A matriz proposta foi nomeada "Forma, Natureza, Função" (FNF), e dessa maneira evidencia-se três características consideradas na proposição do método, tendo o intuito de ser utilizado pelo projetista no início da etapa de projeto preliminar, momento da configuração do leiaute inicial do produto.

Partindo da função, característica pertencente tanto à natureza como aos produtos, o projetista pode observar a matriz FNF e encontrar possíveis soluções naturais de formas otimizadas pela natureza para determinadas funções, podendo empregar essa analogia como um estimulador para a otimização da forma do produto.

O trabalho apresenta inicialmente uma revisão sobre desenvolvimento de produtos com destaque aos objetivos da transição entre o projeto conceitual e preliminar. Na sequência, trata sobre conteúdo da biologia, buscando subsídios para as proposições do trabalho. Ao final, apresenta-se a proposição da matriz FNF, descrevendo sua estrutura e natureza do conteúdo envolvido bem como a forma de sua utilização. 


\section{PROCESSO DE DESENVOLVIMENTO DE PRODUTOS}

O processo de desenvolvimento de produtos consiste na transformações de informações de mercado e negócio em produtos comercialmente viáveis. Esse processo tem sido proposto de várias maneiras, mas em geral apresentam fases comuns, como as de definição do problemas, concepção, configuração, detalhamento e implementação das soluções geradas. Em Back et al. (2008) é apresentado o Processo de Desenvolvimento Integrado de Produtos (PRODIP) como pode-se observar na Figura 1.

O modelo é dividido em três macrofases (planejamento, projetação e implementação) sendo compostas por objetivos, métodos, ferramentas e atividades para contribuir com a equipe de forma sistemática no desenvolvimento de um novo produto ou na melhoria de um existente, com o intuito de obter um resultado final desejado aos participantes do processo, e, posteriormente e principalmente aos usuários do produto.

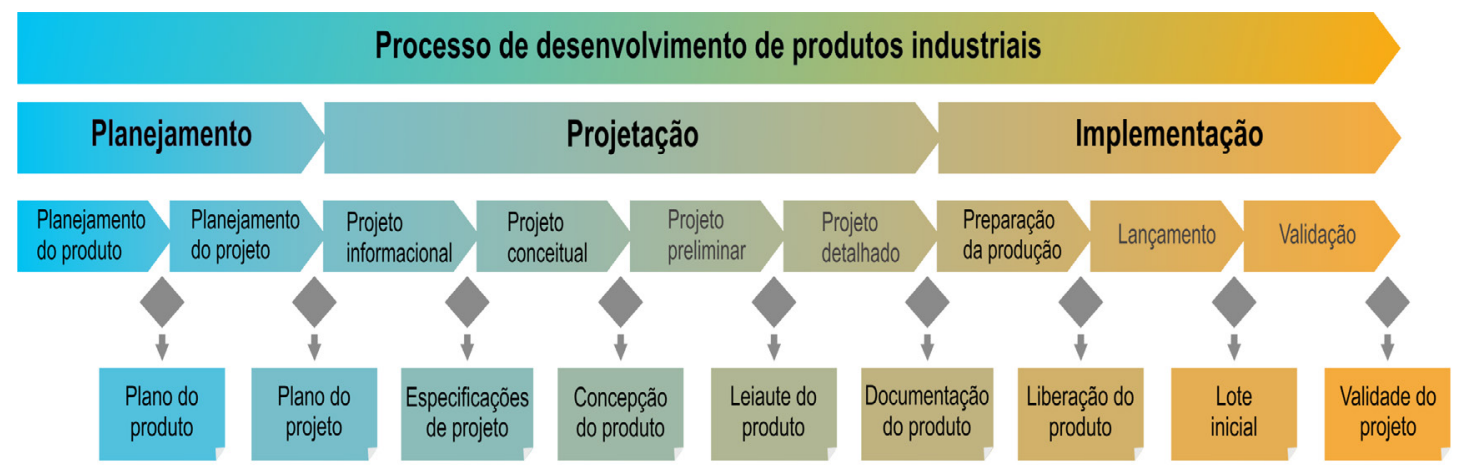

Figura 1 - Processo de Desenvolvimento Integrado de Produtos (PRODIP) Fonte: Adaptado de Back et al. (2008)

A macrofase intitulada planejamento é subdivida em duas fases, sendo: planejamento do produto que está relacionado à busca e seleção de ideias promissoras de produtos, sendo utilizado métodos de ideias como as intuitivas e as sistemáticas, e planejamento do projeto onde são estabelecidos elementos importantes para o gerenciamento do projeto.

A macrofase denominada projetação, conhecida também por processo de projeto, é dividida em quatro fases: projeto informacional onde são desenvolvidas as especificações de projeto; projeto conceitual, em que as soluções conceituais são propostas; projeto preliminar, na qual é desenvolvido o leiaute da solução selecionada e o projeto detalhado, na qual a documentação final do produto testado é finalizada.

A última macrofase do PRODIP é a implementação do produto que está dividida em três fases: Preparação da produção, lançamento e validação. Nessa macrofase é desenvolvido o lote piloto e o lote inicial e o produto é validado junto aos clientes.

Os objetivos do presente trabalho estão relacionados às fases do projeto conceitual e preliminar, as quais serão detalhadas nas seções que seguem. 


\section{PROJETO CONCEITUAL}

A fase de projeto conceitual tem como objetivo final a obtenção de concepção(ões) para o produto que está sendo desenvolvido, buscando satisfazer as especificações de projeto. Para que isso ocorra é necessário inicialmente estabelecer a estrutura funcional do produto.

O método de síntese funcional que foi desenvolvido por pesquisadores como Koller (1985) e Pahl e Beitz (1996) é constituído de função global e possíveis desdobramentos em funções parciais, e caso necessário em funções elementares, conforme pode-se observar na Figura 2.

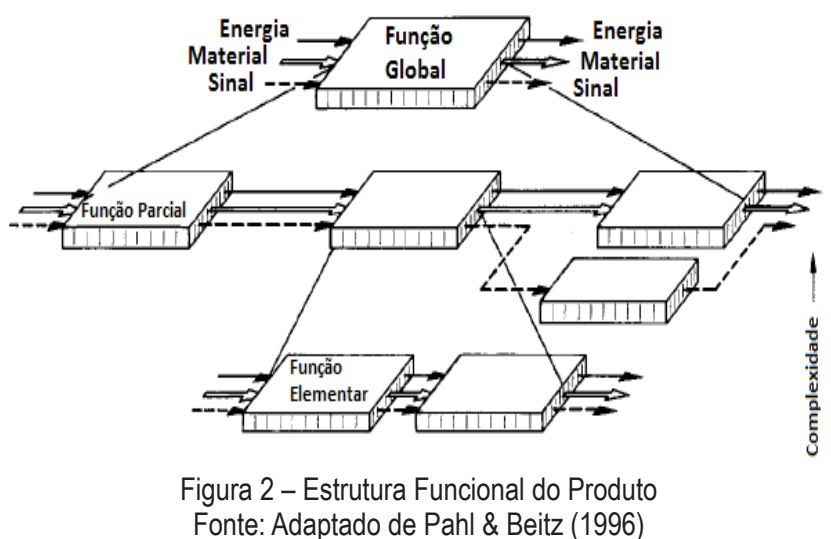

Após a definição das funções e dos seus relacionamentos, a equipe procura por soluções que satisfaçam as funções, buscando satisfazer a função global, que é determinada na fase de projeto conceitual e as especificações do projeto que foram determinadas na fase de projeto informacional.

Para gerar soluções tem-se os métodos que podem ser divididos em intuitivos e sistemáticos. Entre os intuitivos, os mais conhecidos são o brainstorming e as analogias diretas ou simbólicas. Entre os sistemáticos têm-se a matriz morfológica e a análise do valor.

Após a geração de soluções a equipe precisa escolher as melhores, podendo ser utilizado nesse momento o método de Pugh (BACK et al., 2008). Nesse caso devem ser empregados critérios generalizados para avaliar qualitativamente as soluções geradas com relação a uma dada referência.

A solução conceitual selecionada deve agora ser desenvolvida em termos de forma, material, processo, dimensões entre outras características que são objeto do projeto preliminar.

\section{PROJETO PRELIMINAR}

A fase de projeto preliminar é o momento em que a concepção selecionada para o produto "ganha" forma, com o objetivo de satisfazer as especificações do projeto e as funções definidas. Essa etapa inicia-se com o desenvolvimento do(s) leiaute(s) inicial(is) do produto. 
Posteriormente esses precisam ser analisados, geralmente através de modelagem icônica, analógica, numérica ou computacional para verificar se satisfazem o que foi definido.

No final dessa fase a equipe obterá o leiaute final do produto, os materiais a serem utilizados, as definições de componentes padronizados, a consolidação ou necessidade de alteração das principais dimensões, as tolerâncias essenciais ao funcionamento do produto, dentre outros fatores.

\section{TRANSIÇÃO DO PROJETO CONCEITUAL PARA O PROJETO PRELIMINAR}

Após a geração das soluções possíveis e a seleção e combinação das melhores, temse a concepção que irá prosseguir para a fase seguinte, sendo a de projeto preliminar.

Na Figura 3 tem-se um exemplo que destaca a natureza da solução gerada na fase conceitual e preliminar do projeto, destacando a problemática relacionada a esse trabalho, ou seja, como facilitar o trabalho dos projetistas para a geração da forma a partir da concepção desenvolvida?



Figura 3 - Exemplo de soluções conceitual para o preliminar de produto Fonte: Adaptado de (HUBKA, ANDREASEN e EDER, 1988)

O exemplo da Figura 3 apresentado por (HUBKA, ANDREASEN e EDER, 1988) é referente ao projeto de um equipamento para rebitar as sapatas a tambor de freio em veículos. Conforme se observa, cada elemento na solução conceitual foi configurado em termos de forma, dimensões preliminares, materiais e processos.

Esse esboço inicial do produto é geralmente definido pelos conhecimentos prévios dos projetistas adquiridos em sua formação e em projetos desenvolvidos ao longo do tempo, não sendo comum a busca por formas diferentes das conhecidas que poderiam otimizar o produto ou algum componente ou função alternativo.

São poucos os trabalhos que abordam essa transição do conceitual para o preliminar na obtenção de formas, e dentre estes destaca-se o trabalho de Linhares (2005), que usa o conceito de features. Evidencia-se, dessa maneira, a necessidade de uma maior exploração dessa atividade, visando minimizar o retrabalho (tempo e custo) para o melhoramento dos leiautes desenvolvidos. 


\section{BIOMIMÉTICA}

\section{ASPECTOS GERAIS E DEFINIÇÕES}

Dentre os métodos e ferramentas utilizados no processo desenvolvimento de produtos, a analogia com conteúdo da natureza se apresenta como alternativa na obtenção de produtos diferenciados, muitas das vezes relacionados a sustentabilidade e outras com aspectos visuais, podendo ou não se apresentar de maneira otimizada.

A história apresenta que essa relação entre a natureza e o homem na geração de soluções está presente desde épocas primitivas, quando o homem observava situações na natureza e buscava copiá-las, como em ponta de arpões que eram parecidos a ferrões de insetos ou espinhos de plantas (RAMOS; SELL, 1994).

A relação considerada de maneira racional e utilizada em projeto é apresentada em alguns dos principais projetos do italiano Leonardo da Vinci (1412-1519), sendo considerado por alguns pesquisadores como o pai dessa nova ciência. Lobato (2005) menciona sobre o pioneirismo de da Vinci nesse estudo de relação com o mundo natural. Da Vinci fez analogias, tendo início entre o final do séc. XV e começo do séc. $\mathrm{XVI}$, projetando diversas máquinas com analogia a natureza, essas sendo produtos para voo, para aumento da força do homem com a utilização da água, na arquitetura com o corpo humano ou ainda em máquinas de guerra, como o carro blindado, que fez comparação com o formato da carapaça do caranguejo (Figura 4).



A partir da década de 1950 surgiram os conceitos de Biomimética e Biônica como formas de utilizar os conteúdos da natureza na solução de problemas (VINCENT et al., 2006; ANDRADE, 2014). Existem também vários outros termos como Biomimeticismo, Design Inspirado na Natureza, Bioinspiração ou algum tipo de variação da palavra biologia.

Nesse artigo utiliza-se a definição de Biomimética com o intuito de aprender com a natureza, poder imitá-la e aplicar o conhecimento adquirido no desenvolvimento de produtos otimizados. A definição de Biomimética, segundo Geada:

[...] a biomimética visa o estudo da natureza no sentido de aprender com ela (e não sobre ela) e utilizar esse conhecimento em diferentes domínios da ciência. Proveniente do grego "bios", que significa vida, e "mimesis", que significa imitação, a biomimética baseia-se no estudo de sistemas biológicos para desenvolver ou aperfeiçoar novas soluções de engenharia, já que os problemas por eles enfrentados são similares, em muitos aspectos, aos encontrados em sistemas de en- 
genharia. Os biomimeticistas encontram na natureza um modelo perfeito de imitação, de inspiração, pois ela tem muito para nos ensinar, tem muitas respostas às nossas questões. É nesse âmbito, da imitação/inspiração nos modelos/ processos da natureza, que os biomimeticistas procuram respostas com vista a solucionar os seus problemas. (GEADA, 2006)

No decorrer da década de 1990 em diante, a quantidade de estudos que abordam analogia com a natureza é crescente (KNAPP, 2009). Diversas universidades estão trabalhando no aperfeiçoamento dessa ciência, principalmente os departamentos de engenharia, design e arquitetura. Dentre essas, tem-se: Bremen University of Applied Sciences, Rhine-waal University, Jilin University, Arizona State University, Georgia Institute Technology, Havard University, Massachusetts Institute of Technology (MIT), Stanford University, The Ohio State University, University of California - Berkeley, The University of Auckland e Nanyang Technological University.

No Brasil, a biomimética ainda é pouco discutida e estudada, tendo como destaque o laboratório da Escola Superior de Desenho Industrial (Esdi) da Universidade Estadual do Rio de Janeiro (UERJ) e o departamento de Design na Universidade Federal do Rio Grande do Sul (UFRGS).

Dentre as dissertações e teses desenvolvidas no Brasil, tem-se: Ramos (1993), Ramos (2001), Salvador (2003), Allgayer (2009), Sette (2010), Detanico (2011), Neto (2013), Pelegrini (2013), Rosa (2013) e Reis (2013).

A evolução natural dos seres vivos, no qual a espécie humana se inclui, é resultado da necessidade de se adaptar ao ambiente e processo de luta constante pela sobrevivência. A natureza possui milhões de anos de experiência em situações vividas para se adaptar de forma confiável a si própria, dessa forma há infindável conteúdo que pode ser aplicado para inspirar a geração de soluções de projeto.

Detanico (2011) menciona Wahl (2006, p.290) que resgata um ensinamento antigo, do ponto de vista atual, a esse respeito, que a cerca de 500 anos antes, provindo dos diários de Leonardo da Vinci, "aqueles que tomarem partido de qualquer estandarte que não seja a da natureza - a mestra de todos os mestres - trabalharão em vão", evidenciando assim a importância da natureza para otimizar os produtos.

\section{PROPOSTAS PARA USO DE CONTEÚDO DA NATUREZA NA SOLUÇÃO DE PROBLEMAS}

O uso de conteúdo dos elementos presentes na natureza para o desenvolvimento de produtos, principalmente quando a equipe é composta por engenheiros, designers ou arquitetos, e não possuem biólogos, tende a se apresentar logo de início como o primeiro obstáculo para potencializar a obtenção de produtos inovadores e/ ou otimizados. Muitos dos projetistas já descartam essa possibilidade desde o início do processo de solução.

Conforme Eversheim (2009), os conhecimentos sobre os sistemas biológicos podem ser utilizados por meio de quatro formas distintas para o desenvolvimento de soluções para problemas, como se pode visualizar na Figura 5. 


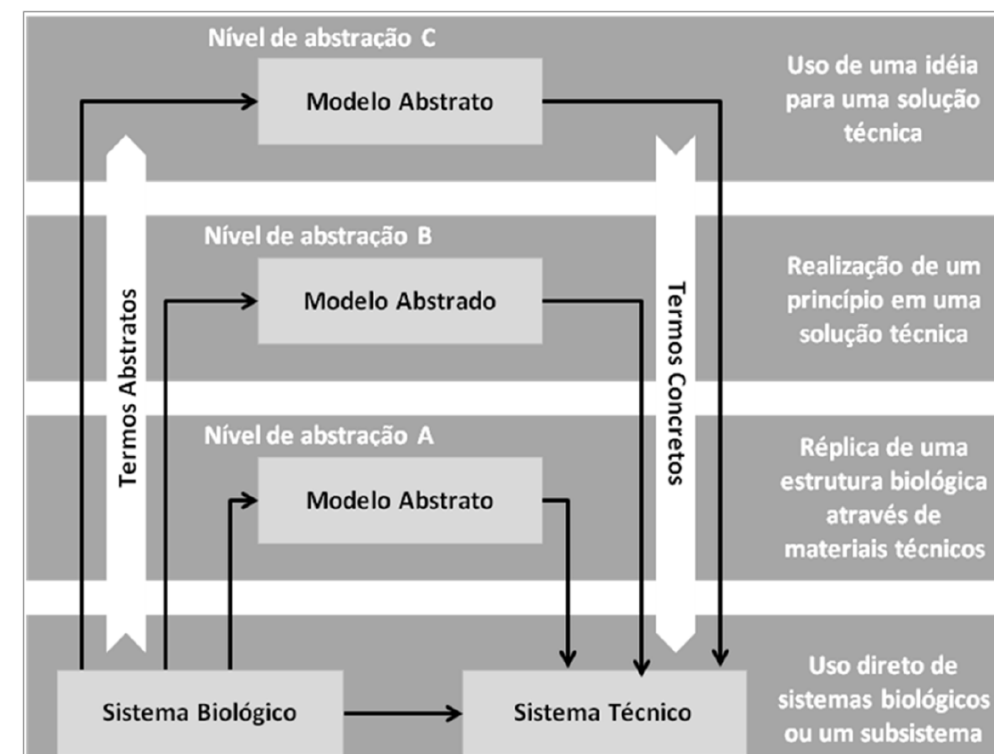

Figura 5 - Possibilidades de utilização dos sistemas biológicos no desenvolvimento de soluções Fonte: (EVERSHEIM, 2009)

Uma das possibilidades é a aplicação direta do sistema biológico. Vários são os exemplos dessa aplicação, tais como aves de rapina treinadas para controlar a presença de outros pássaros em zonas aeroportuárias.

Uma segunda possibilidade (nível de abstração A na Figura 5) é a réplica do sistema biológico por meio de processos técnicos. Nessa categoria incluem-se grande parte das pesquisas em biônica, principalmente na área de design com importância no desenvolvimento de características como aparência, proporções, forma, geometria, entre outras. Como exemplo tem-se réplicas de formas de asas de aeronaves baseada nas asas de pássaros.

De forma mais abstrata tem-se o nível B da Figura 5 que caracteriza-se pela utilização de princípios de solução encontrados em sistemas biológicos, tal como o princípio do sonar empregado pelos morcegos para evitar choques em voo.

O nível $C$ da Figura 5 caracteriza o nível mais abstrato de conhecimento onde os princípios não são usados diretamente mas empregados como estímulo a geração de novas soluções por analogia.

Detanico (2011) também apresenta (Figura 6) caminhos possíveis para desenvolver soluções baseado na natureza procurando facilitar o trabalho de equipes multidisciplinar. 

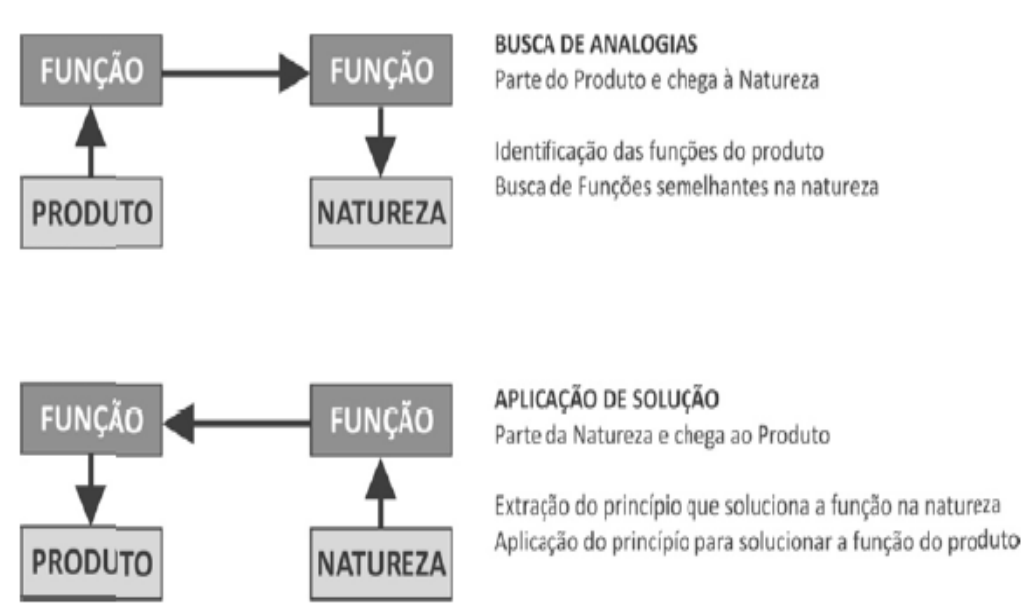

Figura 6 - Analogia entre funções do produto e da natureza Fonte: (DETANICO, 2011)

Na Figura 6 é possível verificar dois caminhos: busca de analogias com a natureza e a aplicação de soluções da natureza. A busca de analogias é a situação em que a equipe está desenvolvendo um produto que tem uma determinada função para resolver. Nesse caso, busca-se na natureza situações com uma função similar. A aplicação de solução é o ciclo inverso, no qual a partir de observações em princípios naturais, verifica-se quais funções são resolvidas e emprega-se o princípio em funções técnicas similares.

Outra proposta desenvolvida por Ramos (1993) é apresentado na Figura 7. O pesquisador sugere que, quando diante de um problema de projeto, a utilização de um banco de dados com soluções presentes na natureza pode facilitar a busca por analogias.

Para o uso de conteúdos da natureza para a configuração de soluções nos produtos, os projetistas precisam pesquisar e encontrar as melhores analogias possíveis. Muitas vezes os mesmos não possuem tempo suficiente para fazer essa busca, ou ainda possuem outros motivos que os impedem, como a falta de conhecimento ou de ferramentas para fazer essa relação. Com isso, acabam desconsiderando formas naturais que poderiam contribuir com a otimização de soluções para o produto.

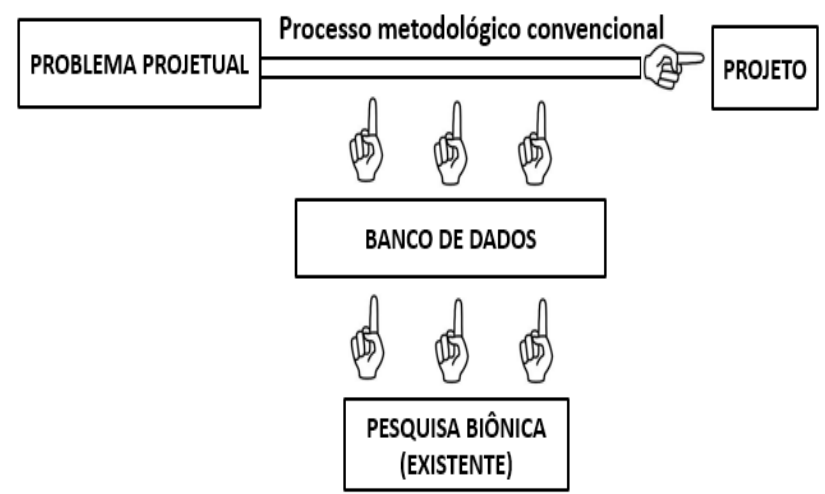

Figura 7 - Banco de dados com princípios de solução da natureza no apoio ao processo de projeto Fonte: (RAMOS, 1993) 
A proposta desse trabalho, por meio de atividades e a matriz FNF tem o objetivo de contribuir com na solução desses problemas, visando facilitar o desenvolvimento de leiautes iniciais dos produtos em direção a soluções otimizadas.

\section{APLICAÇÃO DE CONTEÚDO DA NATUREZA SOLUÇÃO DE PROBLEMAS}

Entre os exemplos conhecidos de uso do conteúdo da natureza para a geração de formas tem-se o projeto da Mercedes-Benz de um carro-conceito com características aerodinâmicas otimizadas.

Para isso, a equipe buscou inspirações na natureza, realizando visita ao museu de história natural de Stuttgart na Alemanha. Entre os peixes observados no museu, como tubarões e golfinhos, um deles foi o peixe-cofre, também conhecido como boxfish. Percebeu-se que o mesmo se movimentava com muita eficiência, tendo aparentando baixo coeficiente de arrasto na água (ALCOFORADO, NASCIMENTO E NEVES, 2013). Além disso pela aparência de seu volume, poderia contribuir com um maior conforto para os passageiros em termos de espaço, tornando-se assim uma potencial forma a ser analisada. Sua forma é similar a uma gota d'água (Figura 8).

Com base nessas características foram propostas soluções para o novo veículo e denominado Bionic Car. O protótipo construído foi testado em túnel de vento e tanques d'água e os resultados foram bastante satisfatórios, com baixo consumo comparado com modelos equivalentes e melhor rigidez estrutural. (MERCEDES -BENZ, 2015; SHARFMAN, 2006).

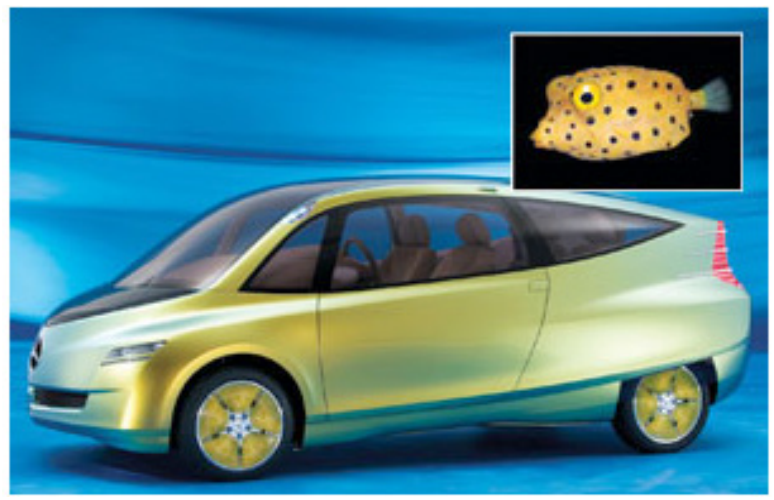

Figura 8 - Bionic Car da Mercedes-Benz e peixe-cofre Fonte: (SHARFMAN, 2006)

\section{MATRIZ FNF PARA AUXILIAR NO DESENVOLVIMENTO DE LEIAUTES INICIAIS DE PRODUTOS}

Os produtos são definidos por meios de suas funções, que podem ser estruturadas em função global, parciais e elementares.

Nessa proposta considera-se as funções dos produtos como o elemento de ligação entre os conteúdos da natureza e os problemas técnicos relacionados ao produto (Figura 9). Assim, propôs-se uma matriz que relaciona soluções da natureza com típicas funções que podem ser utilizadas para representar os problemas de projeto 
na configuração de formas de produtos. Dessa forma, diante de dada função, o projetista orienta-se pelas soluções propostas na matriz, para gerar formas alternativas.

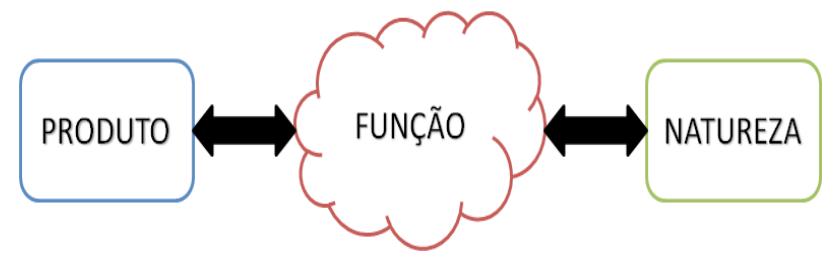

Figura 9 - Relação entre produto e natureza Fonte: Próprio autor

A matriz proposta neste trabalho intitulada Matriz FNF (Forma, Natureza, Função) tem a estrutura mostrada na Figura 10. As colunas são constituídas de informações sobre: função, forma, significado da função, sinônimos - propostos por Koller (1985), outros sinônimos e função inversa a função que foi determinada no início.

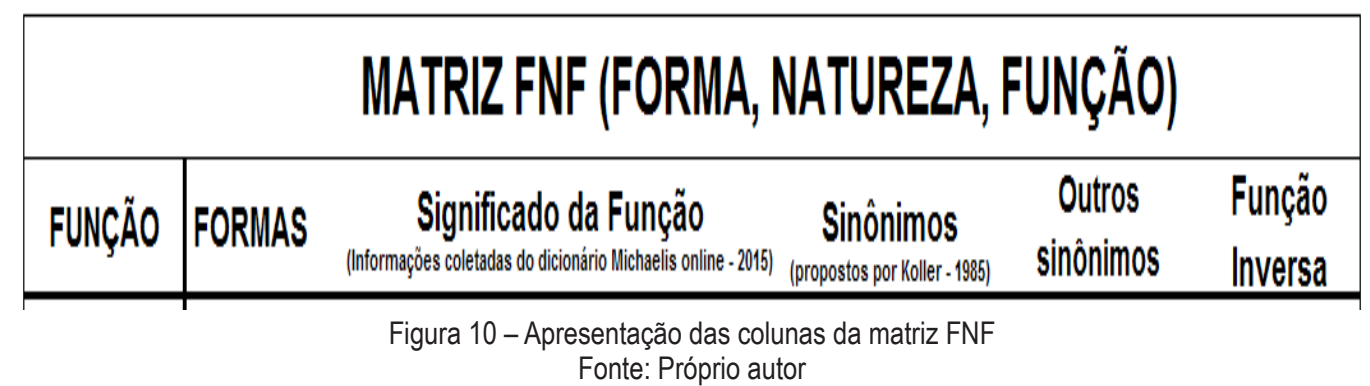

Os elementos presentes na matriz FNF (ver Figura 10) possuem finalidades, sendo: função, o local inicial da utilização da matriz pelos projetistas, devido ser uma informação existente que foi desenvolvida anteriormente na síntese funcional do produto; forma, onde são apresentadas as formas naturais desenvolvidas baseadas em analogias presentes na natureza, sendo que a escolha da forma nesse local faz com que o usuário seja encaminhado para uma ficha (e.g. ver Figura 12); significado da função, é a definição da função, para que não gere dúvidas sobre o seu significado; sinônimos - propostos por Koller (1985), são aqueles que foram propostos pelo referido autor, onde é possível ser verificado na Figura 11; outros sinônimos, tendo relação com a função, com o intuito de aumentar a possibilidade de encontrar a função que está sendo procurada, caso não seja encontrada inicialmente na coluna função e a função inversa, onde é possível encontrar o antônimo da função apresentada inicialmente.

As funções presentes na matriz FNF foram definidas com base nas 24 funções sugeridas por Koller (1985) e adaptadas em Back et al. (2008), como pode se observar na Figura 11. Essas funções representam uma síntese daqueles potenciais para modelar abstratamente os produtos.

Com base no estudo de soluções da natureza, para cada função da matriz foi pesquisado um conjunto de soluções típicas para satisfazê-las. Nessa pesquisa foi criada uma ficha (Figura 12), que representa o conteúdo associado a função e formas que podem ser desenvolvidas. 


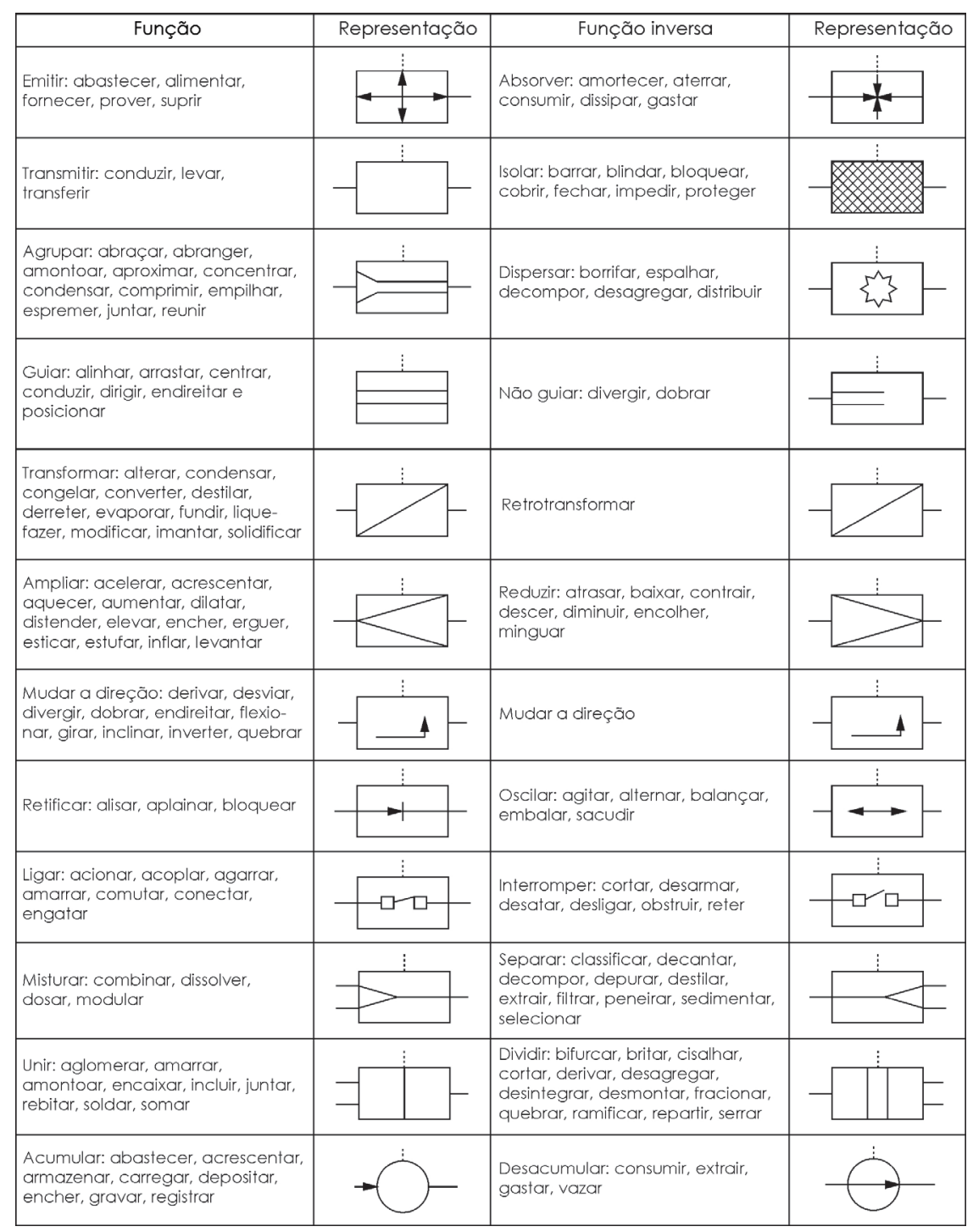

Figura 11 - Funções elementares para representar ações em sistemas técnicos Fonte: (BACK et al., 2008 adaptado de Koller, 1985)

As fichas foram desenvolvidas para facilitar o uso do conteúdo da natureza, possuindo o número que está associado a matriz FNF, a função, a forma, a analogia sugerida, imagem do elemento presente na natureza, esboço, características a se destacar, motivo da analogia sugerida, outras informações e referências.

Para o preenchimento da matriz FNF e das fichas foram analisados materiais acadêmicos que relatam sobre a utilização de elementos da natureza em produtos (artigos, dissertações e teses), empresas que utilizaram a analogia com sucesso e em livros específicos da biologia.

Dessa maneira, a matriz FNF organiza o conteúdo da natureza por meio de um conjunto de funções e sinônimos e serve de referência para, a partir da função que se deseja resolver, buscar conteúdo análogo na natureza que encontra-se sistematizado por meio das fichas desenvolvida. 


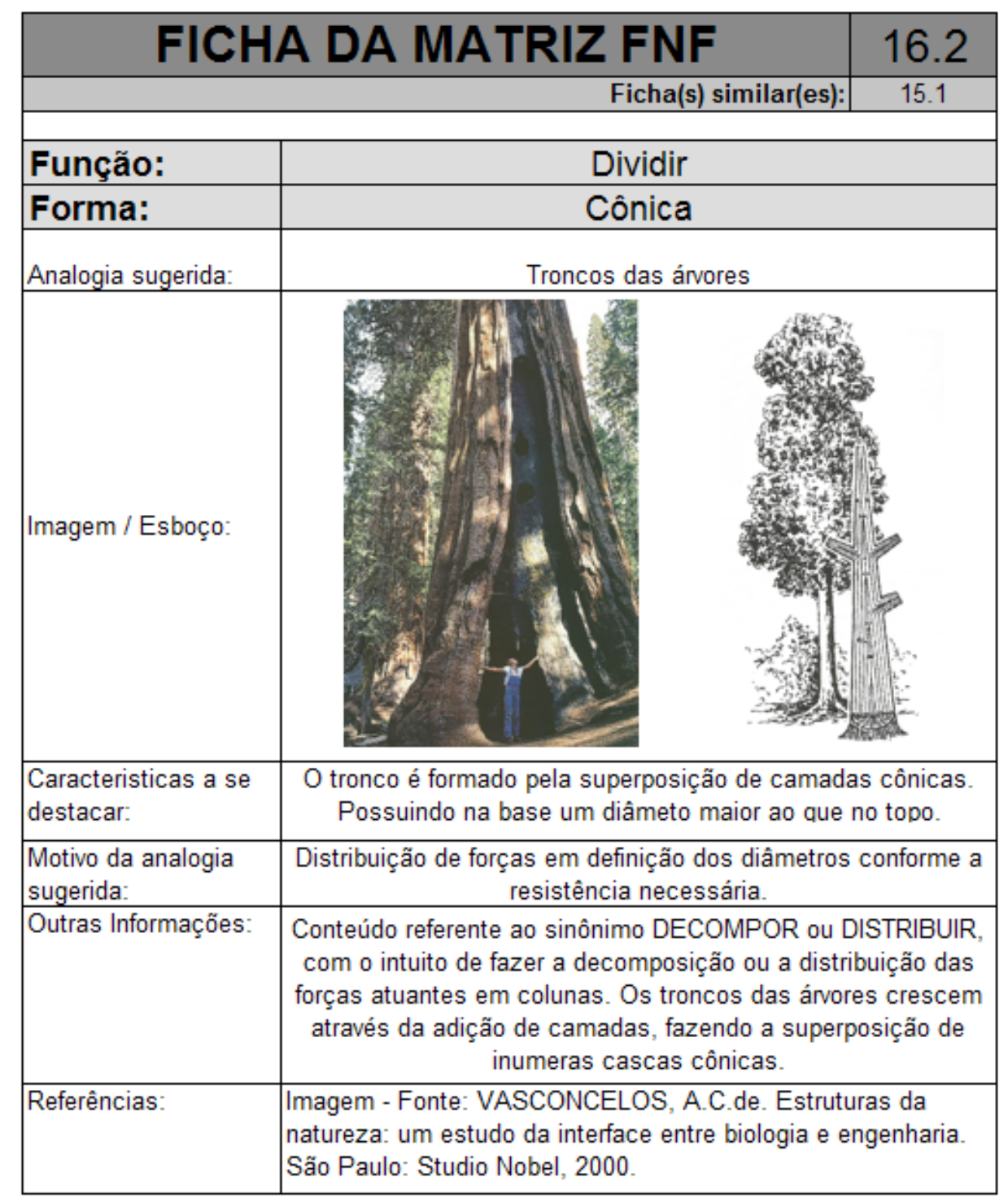

VOLTAR A MATRIZ

Figura 12 - Ficha referente a otimização possível número 16.2 Fonte: Próprio autor

Para ilustrar o uso da matriz FNF tem-se na Figura 13 um extrato das linhas 15 , 16, 17 e 18 da matriz com o preenchimento de uma imagem do tronco das árvores, elefante, formiga, vitória régia, garra das aves, tubarão branco (dentes), patas de gato e tatu-bola. Para cada uma dessas imagens existe uma ficha associada, conforme visto na Figura 12 para o caso do tronco de árvore, com conteúdos correspondentes.

Com isso, o projetista verifica na matriz FNF a função para qual deseja buscar uma forma, e caso não encontre a função desejada, é possível tentar localizar através de sinônimos. Após localizar a função e por consequência os elementos presentes na natureza sugerido na matriz FNF, é possível selecionar e acessar a ficha da matriz FNF, que contêm informações detalhadas sobre a analogia sugerida. Com essas informações o projetista possui orientações organizadas que podem contribuir na geração de leiautes iniciais de produtos. 




A matriz FNF é utilizada por meio de uma sistemática que é composta pelas atividades apresentadas na Figura 14, sendo: 1 - Identificar as funções na concepção, 2 - Definir o escopo funcional, 3 - Selecionar o CBio (matriz + ficha) e 4 - Gerar o leiaute inicial.

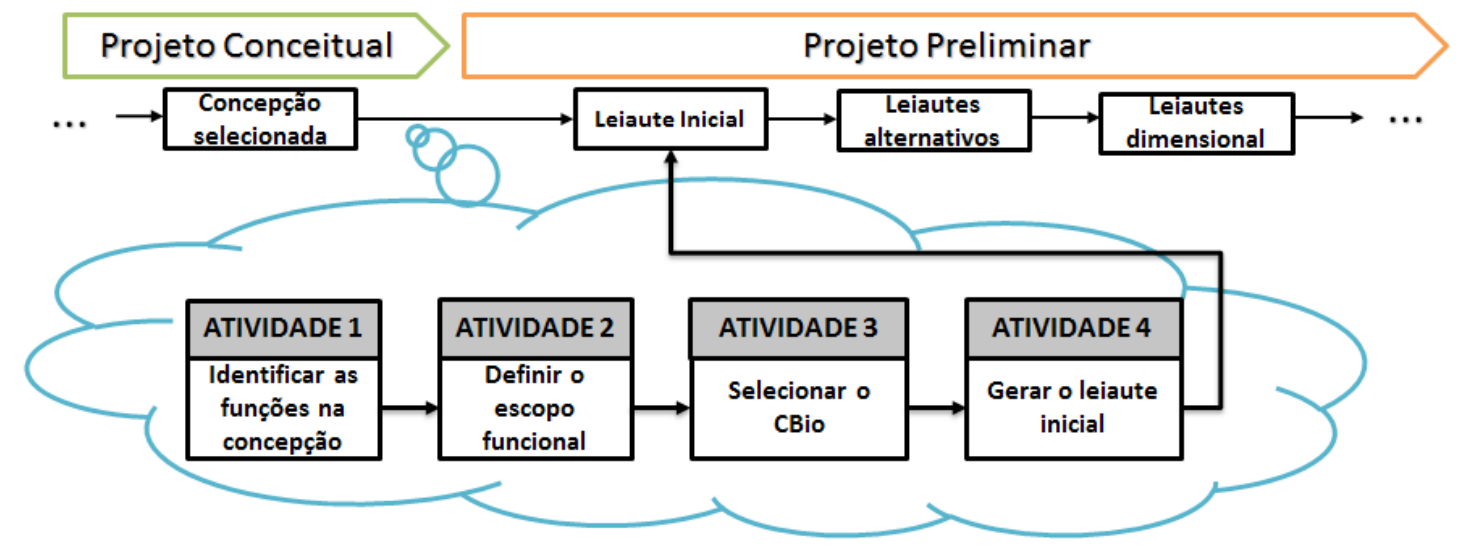

Figura 14 - Sistemática para a configuração da forma de produtos por analogia com a natureza Fonte: Próprio autor

Por meio dessas atividades, num primeiro momento o projetista indica em cada elemento presente no diagrama esquemático da concepção as correspondentes funções. Trata-se da identificação da informação de entrada a utilização da matriz FNF, pois dessa maneira evidência as funções em seus respectivos locais de atuação no diagrama.

Na sequência, é necessário executar a atividade de definição do escopo funcional, sendo o momento de selecionar qual(is) função(ões) deve ter prioridade para buscar a analogia em formas presentes na natureza em relação as outras. Entre os 
métodos utilizados para a classificação das funções, conseguindo dessa maneira verificar as que possuem prioridades, tem-se a votação da(s) função(ões) prioritárias pelos membros da equipe do projeto ou a análise comparativa entre todas as funções presentes no produto, sendo fornecido conceitos para cada comparação realizada, para que posteriormente seja feito o somatório dos conceitos atribuídos para cada função, obtendo no final a(s) função(ões) que possuem prioridade.

$A$ atividade seguinte é a referente a CBio (conhecimentos biológicos), mencionada como a atividade de definir o CBio (matriz + ficha). Essa atividade corresponde ao uso da matriz FNF e as fichas conforme exemplo apresentado na Figura 12. Com base nas funções e fichas identificadas tem-se o conteúdo para desenvolver analogia e buscar soluções para o problema em questão. O projetista pode utilizar uma ou mais de uma ficha para produzir a forma.

Para finalizar tem-se a última atividade que corresponde a definição do leiaute inicial do produto análogo as formas encontradas na natureza e que foram observadas na matriz FNF e nas fichas correspondentes. O leiaute inicial é o primeiro momento que o produto é apresentado pela equipe contendo formas que estão relacionadas com as especificações e as funções definidas anteriormente e que, com a utilização dessa sistemática, possuem relação com conteúdos presentes na natureza.

\section{CONSIDERAÇÕES FINAIS}

O incentivo a amplos estudos de biomimética aplicados a diversas áreas do conhecimento devem ser ampliados para que seu uso se torne frequente e popular em indústrias e escritórios de desenvolvimento de produtos, com o objetivo de oferecer uma alternativa eficaz.

A importância dessa proposta encontra-se na sistematização do conteúdo da natureza em maneiras simples e práticas para a utilização em projeto. Também, na forma de organização e busca dessas informações. O uso do conteúdo registrado serve de inspiração e ainda pode ser melhorado por mecanismos de apresentação e busca da informação.

A transição da solução conceitual para o leiaute inicial é fortemente dependente de conhecimentos prévios do projetista. Assim, para aqueles menos experientes, essa proposta apresenta-se como importante contribuição para auxiliar na definição de formas apropriadas para as funções do produto pela sua facilidade de utilização e natureza do conteúdo proposto.

A matriz FNF encontra-se em desenvolvimento e avaliação dos mecanismos propostos. Foram previstos trabalhos para testar o uso da matriz em problemas práticos de projeto, cujos resultados serão apresentados em futuros trabalhos. 


\section{REFERÊNCIAS}

ALCOFORADO, M. G.; NASCIMENTO, A. D.; NEVES, F. A geometria da natureza: Um estudo da funcionalidade das formas biológicas para aplicação no design. Florianópolis: [s.n.]. 2013.

ALLGAYER, R. Formas naturais e estruturação de superfícies mínimas em arquitetura. Universidade Federal do Rio Grande do Sul. Porto Alegre, p. 157. 2009.

ANDRADE, G. R. Biomimética no design: Aboradagens, limitações e contribuições para o desenvolvimento de produtos e tecnologias. Univesidade do Estado de Minas Gerais. Belo Horizonte, p. 108. 2014.

BACK, N. et al. Projeto Integrado de Produtos: Planejamento, Concepção e Modelagem. Barueri, SP: Manole, 2008.

BOLME, E. The lost notebooks of Leonardo da Vinci. [S.l.]: Castle Falkenstein, 1995.

DETANICO, F. B. Sistematização de princípios de solução da natureza para aplicação no processo criativo de projeto de produtos. Universidade Federal do Rio Grande do Sul. Porto Alegre, p. 191. 2011.

EVERSHEIM, W. Innovation management for technical products. systematic and integrated product development and production planning. Berlin. Germany: Springer, 2009.

GEADA, O. Biomimética: uma nova abordagem na conquista do espaço. Ciência Hoje, 2006. Disponivel em: <http://www.cienciahoje.pt/index.php?oid=28968op=all>. Acesso em: 15 Abril 2015.

HUBKA, V.; ANDREASEN, M. M.; EDER, W. E. Practical studies in systematic design. [S.l.]: Butterworths, 1988.

KNAPP, L. Invenção: Tecnologias e produtos que vão mudar o mundo. Época Negócios, p. 45-52, Julho 2009.

KOLLER, R. Konstruktionsmethode fur der maschinenbau. Heidelberg: Springer Verlag, 1985.

LINHARES, J. C. Uma abordagem computacional baseada na descrição de funções de peças para projeto preliminar de produto. Universidade Federal de Santa Catarina. Florianópolis, p. 1991. 2005.

LOBATO, F. The Nature of Design. Design Management review, p. 56-61, 2005. 
MERCEDES-BENZ. Bionic Car. Mercedes-Benz. Disponivel em: <https://www.mercedes-benz.com.br/institucional/mundo-mercedes-benz/carro-conceito-bioniccar>. Acesso em: 3 Abril 2015.

NETO, J. B. Estudo de elemento da natureza para aplicação em design: Biomimetização da estrutura de ninhos de cacicus haemorrhous. Universidade Federal do Paraná. Curitiba, p. 194. 2013.

PAHL, G.; BEITZ,. Engineering Design: A systematic Approach. Berlim: Spring Verlag, 1996.

RAMOS, J. A biônica aplicada ao projeto de produtos. Universidade Federal de Santa Catarina. Florianópolis, p. 56. 1993.

RAMOS, J. Alternativas para o projeto ecológico de produtos. Universidade Federal de Santa Catarina. Florianópolis, p. 152. 2001.

RAMOS, J.; SELL, I. A biônica no projeto de produtos. 2. ed. [S.l.]: 95-112, v. 4, 1994.

REIS, T. H. Desenvolvimento de texturas cm base em estudos biomiméticos acerca dos pássaros da espécie sicalis flaveola (canário-da-terra) associados ao design emocional. Universidade Federal do Rio Grande do Sul. Porto Alegre, p. 101. 2013.

ROSA, L. C. Desenvolvimento de carenagem para miniveículo visando eficiência energética com base na biônica. Universidade Federal do Rio Grande do Sul. Porto Alegre, p. 114. 2013.

SALVADOR, R. J. Metodologia biônica em dobradiça de móveis. Universidade Federal do Rio Grande do Sul. Porto Alegre, p. 122. 2003.

SETTE, T. C. C. Desenvolvimento de uma proposta de indicadores para avaliação do desempenho ambiental dos processos produtivos industriais sob a ótica da biomimética. Universidade Vale do Rio dos Sinos. São Leopoldo, p. 142. 2010. 